\title{
'Elevated' hemidiaphragm due to a pericardial cyst
}

\author{
V.A.P. Borghouts · Y.J. Stevenhagen · L.J. Wagenaar · D.E. Bouman · P.M.J. Verhorst
}

Published online: 21 December 2015

(C) The Author(s) 2015. This article is published with open access at Springerlink.com

A 60-year-old patient was seen in our gastroenterology outpatient clinic for the evaluation of abnormal liver function tests. Chest X-ray demonstrated what was interpreted as an elevated right hemidiaphragm (Fig. 1). Abdominal sonography revealed a fluid collection above the liver.

A contrast-enhanced computed tomography (CT) scan of chest and abdomen was performed and revealed a nonenhancing, fluid density large supradiaphragmatic mass located in the right cardiophrenic angle, with close relation to the pericardium. Cardiac magnetic resonance imaging (CMR) of the thoracic cavity confirmed the mass to be a $17 \times 13 \mathrm{~cm}$ pericardial cyst (Fig. 2).

\section{Discussion}

With an incidence of approximately 1 in 100,000, pericardial cysts are rare mediastinal masses [1]. Seventy percent of pericardial cysts are located at the right cardiophrenic angle [1, 2]. Most patients are asymptomatic [1, 3], resulting in the diagnosis of a pericardial cyst as an incidental finding on thoracic imaging studies [1-3]. Compression of the heart can cause symptoms resulting in chest discomfort,

V.A.P. Borghouts $(\varangle)$ · Y.J. Stevenhagen · L.J. Wagenaar · P.M.J. Verhorst

Department of Cardiology, Thoraxcentrum Twente, Medisch

Spectrum Twente,

Enschede, The Netherlands

e-mail: v.borghouts@gmail.com

\section{D.E. Bouman}

Department of Radiology, Medisch Spectrum Twente, Enschede, The Netherlands dyspnoea or palpitations [1, 3-5]. In symptomatic patients, percutaneous aspiration and ethanol sclerosis can be suggested, as well as surgical resection $[3,5]$. In asymptomatic patients, no treatment is necessary [5]. Annual observation is optional and can be performed by echocardiography, CT or CMR.

Funding None.

Conflict of interest None declared.

Open Access This article is distributed under the terms of the Creative Commons Attribution License which permits any use, distribution, and reproduction in any medium, provided the original author(s) and the source are credited.

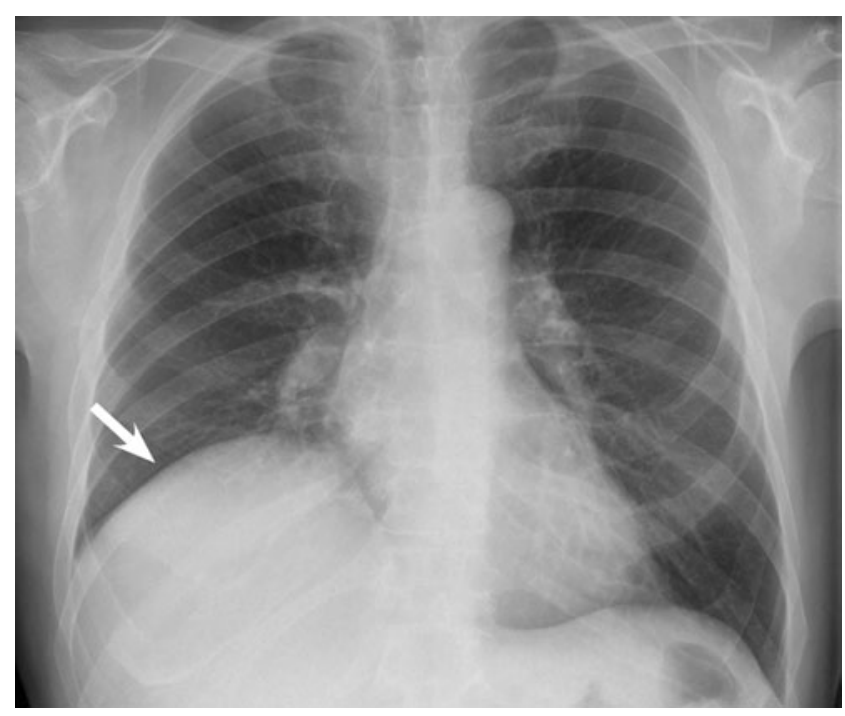

Fig. 1 Upright chest x-ray demonstrating what seemed to be an elevated right hemidiaphragm (arrow). 


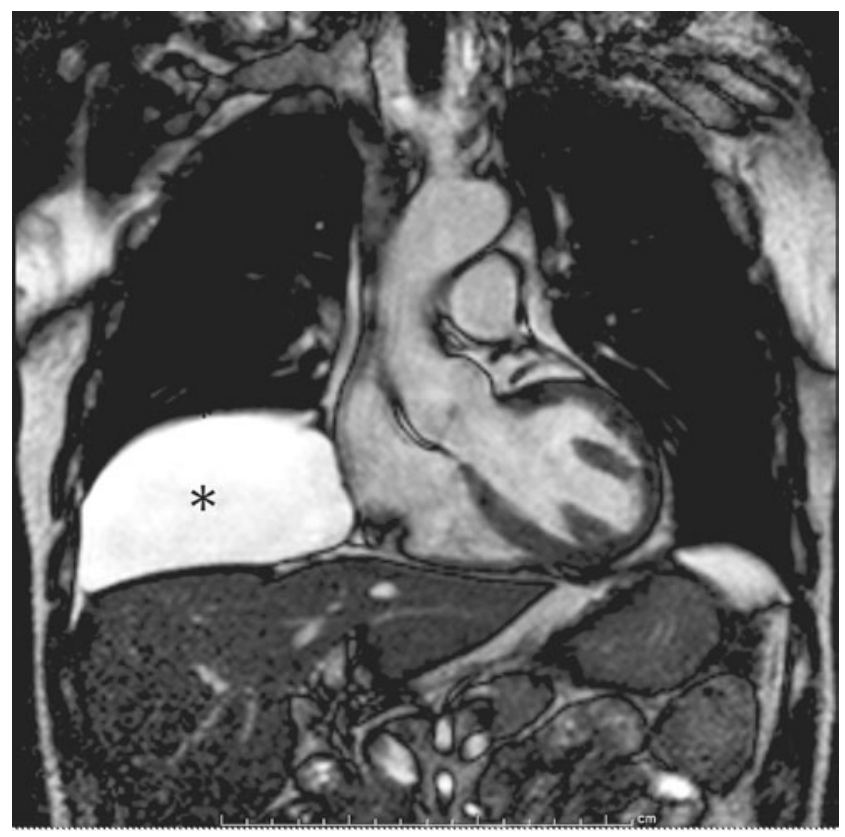

Fig. 2 Cardiac magnetic resonance imaging showing a large mass outside the pericardium, with high signal intensity on T2-weighed imaging, most consistent with a pericardial cyst (asterisk).

\section{References}

1. Patel J, Park C, Michaels J, Rosen S, Kort S. Pericardial cyst: case reports and a literature review. Echocardiography. 2004;21:269-72.

2. Yared K, Baggish AL, Picard MH, Hoffmann U, Hung J. Multimodality imaging of pericardial diseases. JACC Cardiovasc Imaging. 2010;3:650-60.

3. Bezgin T, Elveran A, Varol S, Doğan C, Karagöz A, Esen AM. Pericardial cyst. Herz. 2014;39:1005-7.

4. Kumar Paswan A, Prakash S, Dubey RK. Cardiac tamponade by hydatid pericardial cyst: a rare case report. Anesth Pain Med. 2014;4:e9137.

5. Nayak K, Shetty RK, Vivek G, Pai UM. Pericardial cyst: a benign anomaly. BMJ Case Rep. 2012. doi:10.1136/bcr-03-2012-5984. 